\title{
Mesure de l'exposition aux Composés Organiques Volatils en milieu industriel
}

\author{
J.F Coudert
}

\author{
C\&C IngeScience, 1317, Chemin des Bastions, 06370 Mouans-Sartoux, France
}

\begin{abstract}
Résumé. Les Composés Organiques Volatils (COV) sont des gaz émis par des milliers de produits d'usages courants. Beaucoup d'entre eux sont dangereux pour l'exposition humaine.

De nombreux pays disposent de législations pour contrôler ces expositions.

Il est donc nécessaire de réaliser des mesures pertinentes et fiables de concentrations dans l'air dans différents contextes industriels complexes.

Cet article présente les résultats de nombreuses mesures réelles. Il montre que de telles mesures permettent souvent de trouver des solutions simples et économiques de réduction des expositions.

Il montre également la nécessité d'avoir sur site des capacités d'étalonnage des instruments pour obtenir des résultats fiables.
\end{abstract}

\begin{abstract}
Volatil Organic Compounds (VOC) are gases emitted by thousands of products which are of common use in our environment. Many of them are dangerous for human exposure.

A number of countries are enforcing legislations to control these exposures.

To do so, it is necessary to have pertinent and reliable air concentration measurements in many different and complex industrial contexts.

This paper presents results from many real life measurements. It shows that such measurements help often finding simple and cheap solutions for exposure reduction.

It also emphasis the need for having on site calibration capabilities of instruments to get reliable results.
\end{abstract}

Depuis 2006, C\&C IngeScience a consacré une partie de son activité dans le cadre d'un projet de recherche initialisé avec OSEO-Anvar, portant sur la mesure et l'analyse de l'exposition aux vapeurs chimiques des personnels des industries des parfums, des cosmétiques et des arômes. Cette activité s'est ensuite étendue au secteur agro-alimentaire.

Développé en relation avec un industriel, ce projet comportait 2 volets

Le premier volet concernait la mise au point d'un logiciel permettant :

- de décrire le processus de production d'un atelier de compostions, avec les flux de matières premières et les opérations qui conduisent à une exposition des personnels (pesées, soutirages, agitations, transvasements...),

- de dénombrer quotidiennement ces opérations et de leur associer les quantités manipulées,

- de compiler les tuples (opérateur, matière_première, quantité, opération),

- De renseigner et d'exploiter une base de données avec ces informations,.

Cet objectif a conduit à la réalisation d'un logiciel prototype «ABH-Protect » (Fig.1).
Le deuxième volet concernait la mise au point et la validation d'une méthode de mesure des profils d'expositions en temps réel sur les postes de travail permettant :

- d'identifier les sources et les contextes entrainant une exposition (disposition du poste, posture et gestes techniques de l'opérateur) pour une opération donnée,

- de quantifier les expositions en fonction de l'opération et de la matière première ou du mélange manipulé,

Ce volet a été conduit en relation avec les conseils techniques du Laboratoire de Chimie de la CRAM Sud-Est. Il s'est ensuite poursuivi pour le compte d'autres industriels.

L'objet de ce document est de présenter ce volet « Mesure de l'Exposition ».

\section{Identification des sources d'exposition, mesure des concentrations inhalées, profils d'exposition}

La problématique n'est pas de mesurer l'ambiance d'un atelier ou l'exposition moyenne pendant 8 heures mais bien d'identifier et de quantifier les profils d'exposition liés aux nombreuses opérations réalisées quotidiennement.sur chaque poste de travail (mesures localisées).

Une première difficulté de mesures est liée essentiellement au caractère transitoire de ces expositions, 
typiquement plusieurs secondes à quelques minutes (Fig.2 à Fig. 5). Ceci rend impossible l'utilisation de méthodes lentes de prélèvements nécessitant plusieurs dizaines de minutes (remplissage de sacs, exposition de fibres ou de tubes adsorbants) pour localiser et identifier les variations. L'utilisation de capteurs portables à photo-ionisation - PID de marque mimiRAESystems, dont le temps de réponse est de l'ordre de la seconde, permet de résoudre cette difficulté. La portabilité des PID permet d'établir des profils d'exposition directement pendant les opérations en se plaçant au voisinage des voies respiratoires $(<30 \mathrm{~cm})$. Elle permet également de localiser des fuites dans les installations. La pertinence de cette approche est reconnue par une étude récente publiée par l'INRS [1].

Une deuxième difficulté est liée à la nécessité d'étalonner les PID pour chaque gaz (agent chimique ou mélange). En effet, le coefficient multiplicateur de la lecture (ppm équivalent Isobutylène) varie dans une large gamme $(0,1$ à 40) par rapport à l'Isobutylène utilisé pour l'étalonnage du PID. Les mesures ne peuvent donc pas être interprétées en valeurs absolues de concentrations sans connaître ces coefficients.

Une troisième difficulté est liée au caractère non sélectifs des PID lorsque les mesures concernent des mélanges d'agents chimiques, cette difficulté se révélant être un avantage pour la mesure des expositions mais imposant des mesures analytiques complémentaires.

\section{Nécessité de pouvoir étalonner les PID}

Les fabriquants de PID fournissent les coefficients d'étalonnage par rapport à l'Isobutylène pour environ deux cents agents chimiques d'usage courant dans la grande industrie [2]. Par contre ces coefficients ne sont pas connus pour de nombreux agents chimiques utilisés dans les industries chimiques spécialisées comme les parfums, les arômes, les encres, les détergents, les colles, etc. . Ils ne sont pas connus non-plus pour :

- les agents chimiques dont la pureté en usage industriel (présence d'autres agents chimiques) est différente de la pureté de l'agent chimique de laboratoire utilisée par le fabriquant du PID pour les étalonnages, les impuretés présentes pouvant modifier sensiblement le coefficient d'étalonnage.

- les mélanges spécifiques contenant plusieurs COV, pour lesquels certains coefficients ou certaines concentrations ne sont pas connus, ceci empêchant l'utilisation de la formule de calcul [2].

- les mélanges dont la composition varie d'une origine à l'autre (ex. : essences naturelles, produits de distillation, carburants...).

- Les mélanges dont la composition est complexe, avec seulement une liste partielle de constituants identifiés

Un dispositif d'étalonnage portable à partir d'un prélèvement de l'agent chimique ou du mélange mesuré a été mis au point par C\&C IngeScience et breveté.

Le principe du dispositif d'étalonnage consiste à créer un échantillon gazeux du COV ou du mélange de COV dans un gaz neutre (ex. azote) avec une concentration connue, dans un sac en TEDLAR (matériau très peu perméable aux gaz sur des durées de quelques heures).
La probématique est liée à la maîtrise de la vaporisation du liquide (COV ou mélange de COV) qui doit être totale dans le sac.

Ces difficultés sont dues à la physique très complexe des phènomènes mis en jeu lors de la vaporisation. Ces phénomènes s'apparentent à ceux rencontrés pour l'optimisation des systèmes d'injection dans les moteurs thermiques. Après de nombreux échecs, un dispositif satisfaisant a été mis au point et testé sur une gamme de COV. La mesure de la concentration connue dans le sac par le capteur comparée à la valeur qu'il affiche permet de calculer le coefficient d'étalonnage propre à l'agent chimique ou au mélange.

Des validations du dispositif ont été effectuées pour comparer les coefficients d'étalonnage obtenus lors de 3 étalonnages successifs réalisés pour différents $\mathrm{COV}$, aux coefficients fournis par le constructeur du PID.

Il est prévu de réaliser prochainement un ensemble de tests complets de ce dispositif avec un laboratoire de référence.

\section{Analyse des mesures réalisées}

Plus de 400 mesures d'expositions portant sur plus de 200 agents chimiques, mélanges et effluents ont été réalisées depuis 2007 dans des entreprises différentes (parfums, arômes, agroalimentaire).

L'analyse des mesures en relation temporelle avec les observations sur les postes de travail fait apparaître plusieurs résultats :

- Il existe une relation directement interprétable entre les pics d'expositions et les postures et les gestes techniques pour la grande majorité des postes de travail. Les 4 exemples représentées sur les Fig. 4 à 7 montrent une corrélation parfaite entre les gestes et les pics d'exposition, y compris pour des opérations aussi élémentaires que des jets de pipettes. L'analyse montre en outre une relation directe avec l'énergie cinétique associée au dispositif ou au geste (ex. hauteur de chute pour un soutirage ou un remplissage, vitesse d'agitation, pression sur la pipette, rapidité du geste technique, etc.). Cette approche débouche sur une prise de conscience des opérateurs et une amélioration directe des gestes techniques.

- Un ensemble de dispositifs ou de dispositions simples sur les postes de travail permet souvent de réduire considérablement les expositions, limitant également l'utilisation d'Équipements de Protection Individuelle (EPI) aux seules phases nécessaires et améliorant ainsi leur appropriation.

- L'efficacité ou le manque d'efficacité de certains dispositifs d'aspiration ont pu être démontrées (circulation générale et recirculation des systèmes d'aspiration, positionnement des bouches d'aspiration fixes ou « cobras », conception ou utilisation de hottes en relation avec l'ergonomie du poste de travail). Un contrôle périodique et fréquent est facile à mettre en place.

Concernant les expositions liées à des interventions ponctuelles en ambiances complexes contenant plusieurs dizaines voir centaines de $\mathrm{COV}$ (stockage chambres froides 
ou chaudes, effluents provenant de traitement sur des matières naturelles), il est nécessaire d'associer aux mesures temporelles un prélèvement à fin d'analyses GC/MS ou LC/MS ultérieures. Ce prélèvement peut être réalisé en parallèle ou en sortie du PID. Elle a déjà été mise en œuvre en paralélle à l'utilisation des PID dans le cadre de travaux sur la caractérisation des pics d'exposition [4]. La mise en œuvre en série avec le PID semble plus rigoureuse dans la mesure où le prélèvement voit exactement les mêmes molécules que le PID. En effet, selon le fabriquant du matériel, les molécules ne sont pas altérées par le passage dans le PID. Elle n'est cependant utilisable que si les relations débit-durée sont rendues compatibles entre elles pour les différents matériels.

\section{COUPLAGE PID- PRÉLÈVEMENT- ANALYSE GC/MS}

Les considérations précédentes ont donné lieu à plusieurs expérimentations basées sur l'utilisation de dispositifs de prélèvements différents en aval du PID (Fig.6).

Ces expérimentations ont montré les difficultés rencontrées dans différentes entreprises, par exemple :

- Mesures réalisées dans une chambre froide stockant environ $200 \mathrm{COV}$. Le prélèvement est réalisé en sortie du PID par barbotage dans de l'eau pure pendant 30 minutes. L'analyse de l'eau par adsorption par un twister n'a pas permis de quantifier les COV.

- Mesures réalisées sur un effluent de presse de matières naturelles (extraction). Le prélèvement par fibre SPME a permis d'identifier un grand nombre de COV mais la quantification par LC/MS est restée peu fiable.

- Mesures réalisées sur le process précédent par prélèvement dans un sac TEDLAR pendant 20 minutes. Le but recherché était d'identifier et de quantifier les composés soufrés présents dans l'effluent. L'analyse des sacs TEDLAR a été réalisée 24 heures après le prélèvement pour des raisons de transport vers le laboratoire d'analyse distant. L'analyse a été réalisée par la Société Explorair avec une micro-GC/MS directement branchée sur le sac. La méthode a permis d'identifier et de quantifier les composés soufrés mais a montré sa fragilité (décroissance de la charge en COV dans les sacs). La mesure du contenu du sac avec le PID au moment de l'analyse GC/MS a permis, par comparaison avec celle réalisée lors du prélèvement, d'estimer cette perte par rapport au contenu initial. Ces résultats semblent inhérents à l'utilisation des sacs de prélèvement en général lorsqu'ils sont conservés des dizaines d'heures. Il est clair qu'un prélèvement dans un sac avec une pompe d'aspiration non contrôlée par un PID ne permet pas d'estimer la perte de contenu et que le résultat de la mesure peut être alors erroné.

- Mesures réalisées sur le process précédent par prélèvement au travers de tubes CARBOGRAPH pendant 10 minutes. Ces tubes ne permettaient pas de mesurer les composés soufrés mais visaient a identifier et quantifier les autres COV présent (plusieurs dizaines). La méthode est apparue robuste.
L'utilisation d'une méthode couplée PID-PrélèvementAnalyse GC/MS ou LC/MS apparaît comme très pertinente pour la mesure, l'identification et la quantification des COV Sa mise en œuvre sur le terrain est simple, souple et beaucoup moins coûteuse que l'utilisation d'une GC/MS transportable sur site dont les temps de réponse ne sont pas compatibles avec la corrélation aux gestes techniques et qui, par ailleurs, n'est pas possible en zones ATEX.

Le choix du couplage (barbotage-Twister, Fibre SPME, sac TEDLAR, Tube CARBOGRAPH) dépend des constituants à analyser, des moyens et de l'expérience du laboratoire d'analyse.

Ce couplage nécessite cependant une préparation en laboratoire pour choisir entre les différents dispositifs de prélèvements (sacs, fibres, tubes) en fonction des COV potentiellement rencontrés et des contraintes de mise en œuvre sur site.

L'approche couplée permet une avancée importante pour la mesure objective des expositions sur des postes de travail et des sites manipulant des mélanges de compositions inconnues, comme par exemple dans les process mettant en œuvre des produits naturels.

\section{SYNTHĖSE DES RÉSULTATS OBTENUS SUITE AUX MESURES}

Les campagnes de mesures réalisées ont permis notamment :

- De faire évoluer une stratégie de maintenance périodique sur une boucle-réacteur-chimique, en localisant les points où l'intervention est particulièrement nécessaire et a mis en évidence la sensibilité de serrage des brides même avec des joints neuf. La réalisation de maintenance sous contrôle de mesures s'est montrée efficace et économique.

- De mettre en évidence la nécessité d'installer des systèmes d'aspirations temporisées préalables à l'ouverture des portes pour les espaces de stockage clos (frigos, chambres froides, etc.).

- D'évaluer l'efficacité des hottes en fonction des morphologies et des positions des opérateurs, ainsi que lors d'ouvertures de portes ou de fenêtres dans le local. L'adoption de dispositions adaptées est en général préférable à la solution d'augmenter l'aspiration en raison de son influence sur les pesées.

- De modifier la ventilation générale d'un sous-sol, en raison de l'interférence extérieure entre les points d'aspiration d'air frais et les points de rejets d'air chargé.

- D'équiper des paillasses dans un laboratoire de recherche avec un système d'aspiration, certains montages expérimentaux pouvant présenter localement et occasionnellement des fuites de solvant.

- De mieux gérer les poubelles et le nettoyage des paillasses.

- D'identifier et de quantifier les bouffées d'effluent lors de l'ouverture de trappes d'inspection sur un process d'extraction de végétaux.

- De classifier les opérations dans un atelier de composition en fonction de leur profil d'exposition (pesées, remplissages, agitations, etc.) et d'identifier les gestes les plus exposants. 
- De constater que la durée d'exposition déterminée à partir du signal mesuré diffère de celle obtenue avec un chronomètre à partir des gestes techniques et n'est pas bien corrélée avec la quantité manipulée. Elle dépend fortement de l'énergie cinétique associée à l'opération.

- De constater que les profils d'expositions sont caractérisés par des successions de pics en relation identifiables, la plupart du temps, avec les gestes techniques. L'identification par l'opérateur des postures et des gestes entrainant le plus d'exposition permet de limiter souvent considérablement celle-ci.

- De constater que les Valeurs Limites d'Exposition Professionnelles (VLEP) courts termes calculées par intégration à partir de mesures comportant de nombreux pics ne sont pas représentatives de la réalité de l'exposition [3][4][5]. Plusieurs pics pourraient même dépasser des valeurs léthales tout en respectant ces valeurs. Pour des Agents Chimiques Dangereux (ACD), la mesure nécessite donc une analyse transitoire.

Des exemples de mesures sont présentés sur les Fig.4 à Fig.7.

\section{Conclusions}

D'une manière générale, la mesure opérationnelle des profils d'exposition a permis d'identifier les sources, les

\section{Références}

[1] : Bruno Galland, Victor Carvhalo, Patrick Martin, Evaluation des detecteurs à photo-ionisation embarqués dans les détecteurs de gaz portables multigaz, INRS- Hygiène et Sécurité du Travail, Cahier de Notes Documentaires, HST-ND2293-212$08,3^{\text {ème }}$ trimestre $2008 \mathrm{p} 212 / 25$,

[2] RAE Systems, Correction Factors, Ionization Energies and Calibration Characteristics, Technical Note TN106 postures et les gestes qui en sont à l'origine. Elle s'est montrée extrêmement efficace pour réduire voir éliminer ces expositions par un ensemble de dispositions matérielles simples et de modification des postures et des gestes techniques en relation avec une prise de conscience des opérateurs.

Elle revêt un intérêt considérable en termes de formation des opérateurs (appropriation, maîtrise des risques).

Elle constitue une étape essentielle pour la préparation des audits réglementaires.

D'autre part la notion de pics d'exposition répétitifs devrait faire l'objet de recherches en toxicologie [4][5]. Les VLEP court termes ne semblent pas bien adaptées à cette réalité.

\section{Remerciements}

$\mathrm{C} \& \mathrm{C}$ IngeScience remercie les directions et personnels des sociétés Jean NIEL SA et Cargill Flavor Systems (actuellement Kerry) à Grasse pour avoir permis et accompagné ces travaux.

La société remercie également Monsieur Jacques Catani, Expert de la CRAM Sud-Est, pour ses conseils et Mademoiselle Asma Rachdi, étudiante en alternance du Master FOQUAL de 1'Université de Nice-Sophia ${ }^{\circ}$ Antipolis, pour sa participation enthousiaste et efficace.

[3] INRS Metropol, Commentaires sur les valeurs limites professionnelles-Définitions et aspects métrologiques, Fiche A2/V01-1/12/2005

[4] Liesbeth Preller, Igor Burstyn, Nettie de Pater, Hans Kromhout, Characteristics of Peaks of Inhalation Exposure to Organic Solvents, Annals of Occupational Hygiene Advance Access, July 27, 2004

[5] Committee on Peak exposure to organic solvents, Peak exposure to organic solvents. Health Council of the Netherland The Hague 1999 pub.n 1999/12

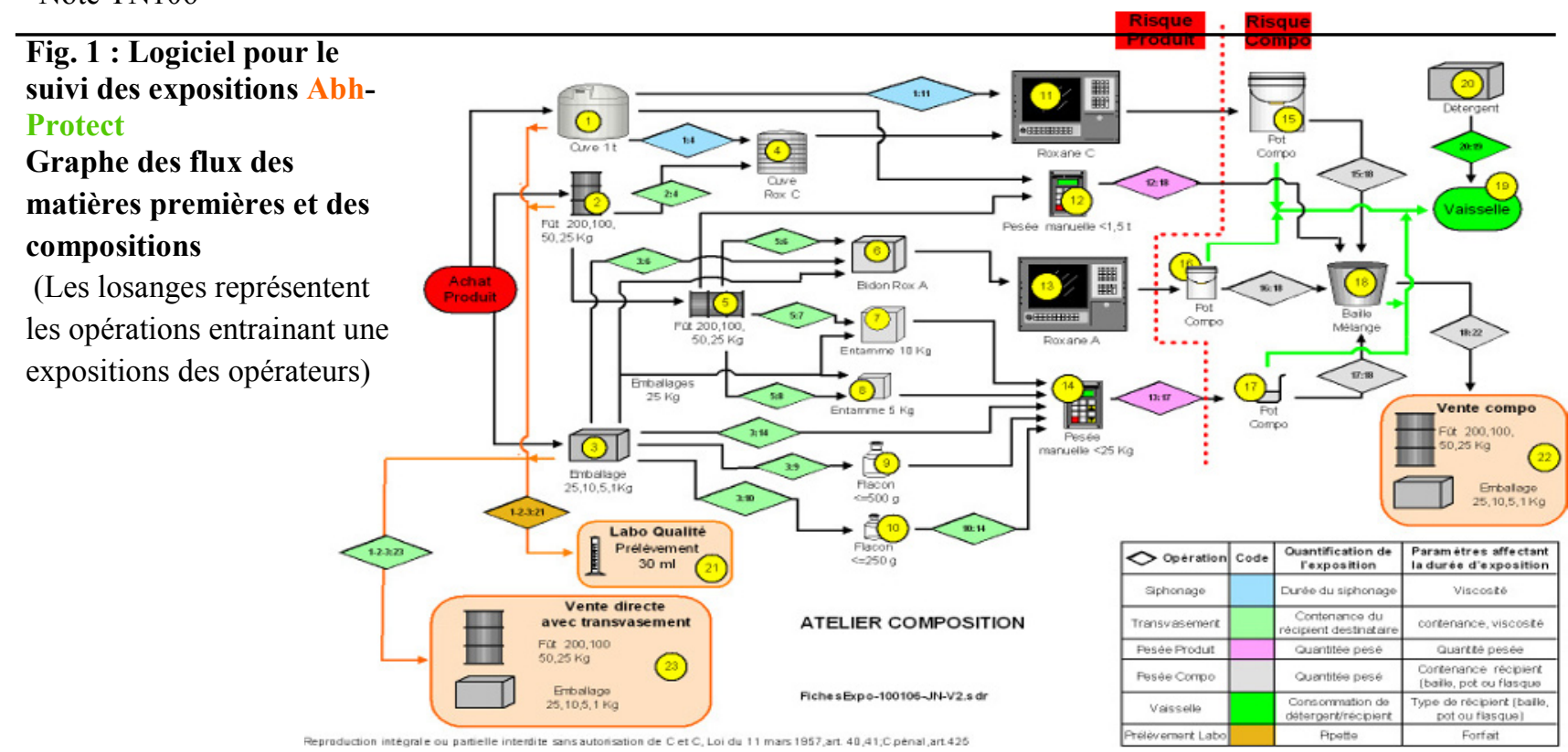




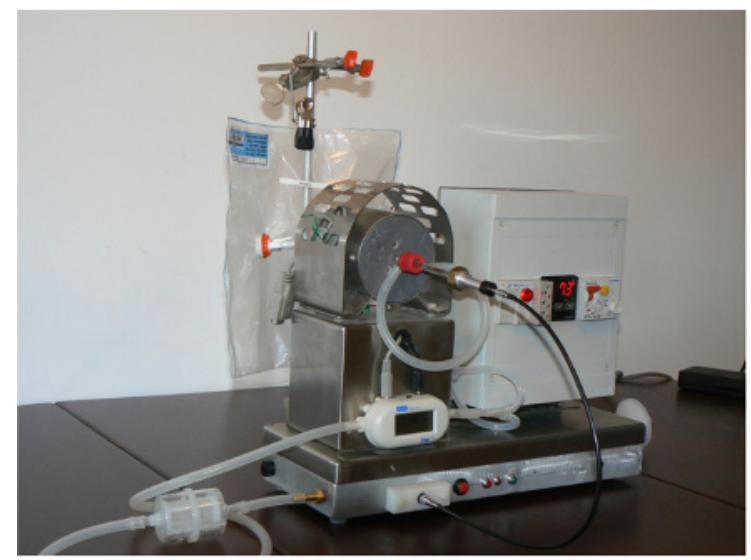

Fig. 2 : Appareil pour réaliser des sacs TEDLAR étalons

Brevet C\&C IngeScience

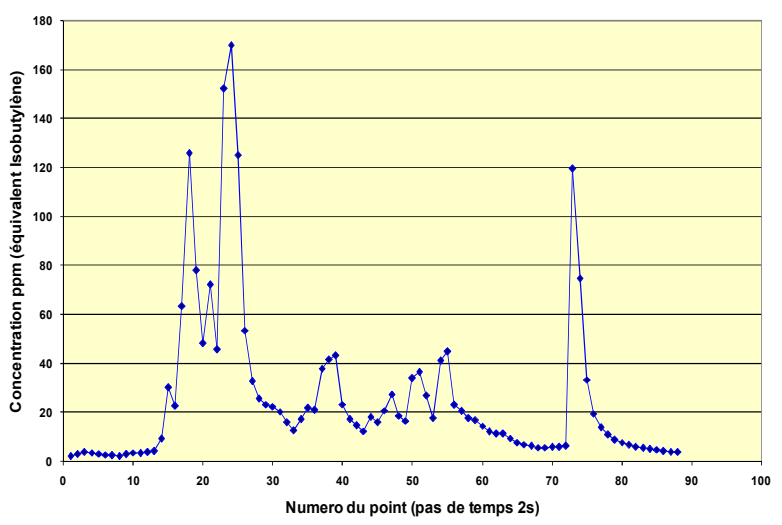

Fig. 4 : Exemple de mesure d'exposition lors d'un remplissage avec pesée d'un réservoir d'un robot à partir d'un fût couché

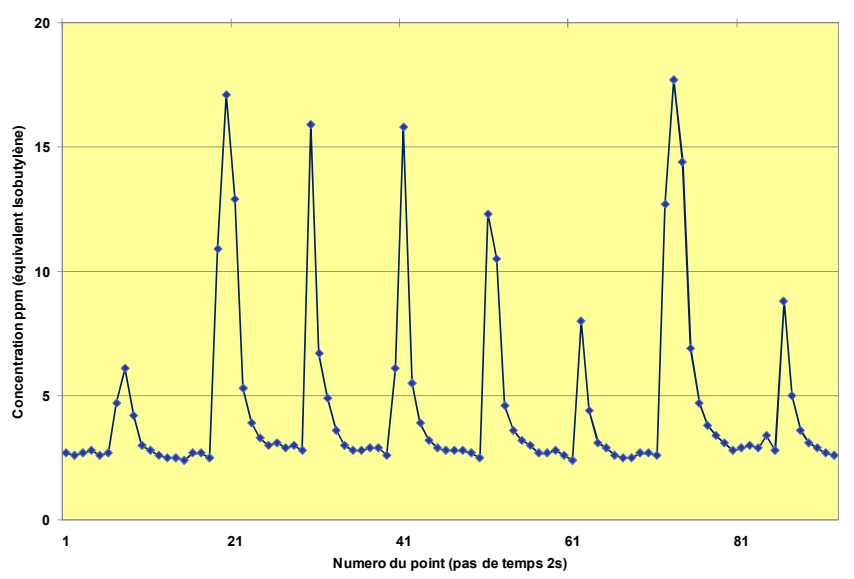

Fig. 5 : Exemple de mesure d'exposition lors d'une pesée à l'aide d'une casse

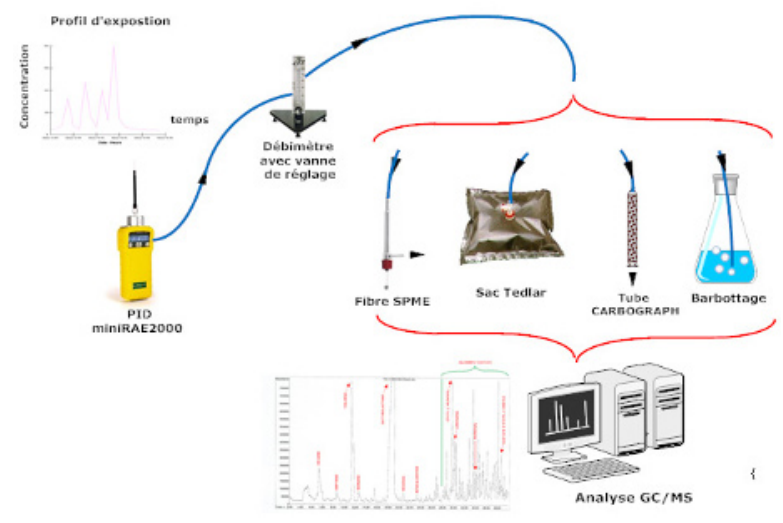

Fig. 3 : Couplage avec différents dispositifs de captage et analyse LC/MS ou GC/MS

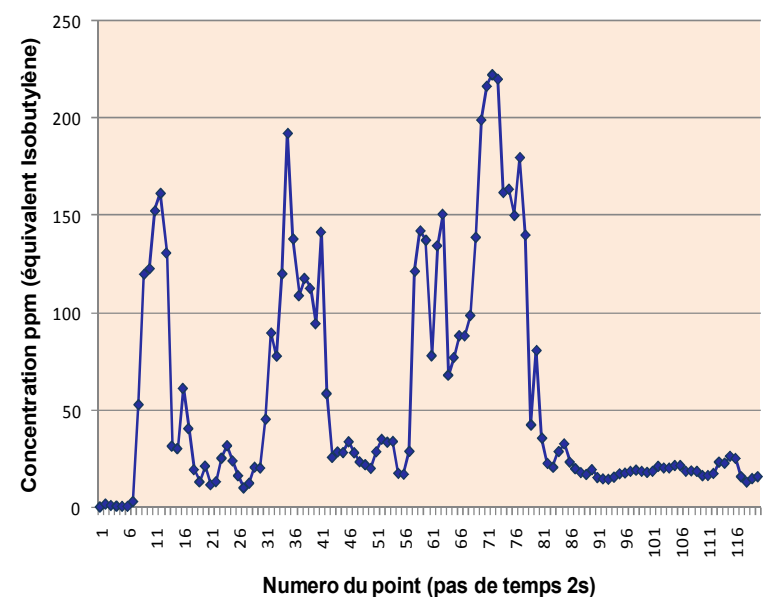

Fig. 6 : Mesure d'exposition lors d'une opération de maintenance d'un filtre à 3 plaques

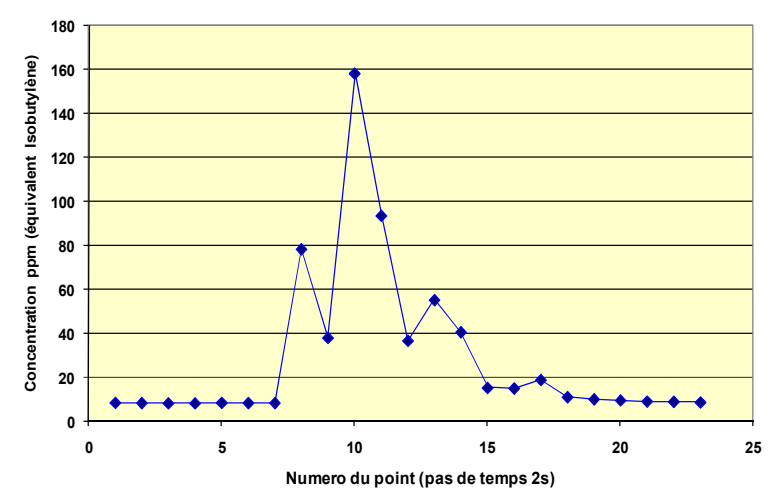

Fig.7 : Exemple de mesure d'exposition lors d'une pesée avec une pipette 